\title{
EI cibercortejo en la adolescencia: riesgos e impacto emocional de la ciberconducta sexual
}

\author{
Virginia Sánchez Jiménez*, Noelia Muñoz-Fernández y Esther Vega Gea \\ Universidad de Sevilla
}

(Recibido 10 Abril, 2015; Aceptado 22 Junio, 2015)

\begin{abstract}
RESUMEN: Las TIC han revolucionado los ecosistemas relacionales humanos, especialmente entre los adolescentes. Partiendo del modelo de la Co-construcción entre los contextos online y offline, es de esperar que adolescentes y jóvenes expresen en ambos escenarios las tareas del desarrollo propias de su etapa evolutiva, como la sexualidad y el inicio de las relaciones de pareja. Estas tareas de desarrollo se convierten en experiencias de aprendizaje fundamentales, si bien no exentas de dificultades, pudiendo derivar en conductas agresivas y de riesgo, como las ciberagresiones sexuales o el sexting. La atención al fenómeno de las agresiones y riesgos de la ciberconducta sexual se ha incrementado en los últimos años, aunque todavía no existen datos concluyentes. Este trabajo se ha planteado describir la prevalencia de estas ciberconductas en la adolescencia tanto en los iguales como en la pareja, así como el malestar emocional percibido. 268 adolescentes con experiencia sentimental (edad media 14.22, d.e. 1.44), fueron entrevistados utilizando una versión adaptada del Sexual Harassment Survey. Los resultados mostraron que estas ciberconductas estaban igual de presentes en las parejas y entre iguales, y que los comportamientos más frecuentes fueron los intercambios de mensajes e imágenes con contenido sexual visual y verbal. Los chicos afirmaron realizar estos comportamientos más que las chicas, pero ambos afirmaron recibirlas en los mismos porcentajes. Las chicas percibieron más molestos estos comportamientos que los chicos. Los resultados se discuten atendiendo al significado que estos comportamientos tienen en la vida relacional adolescente.
\end{abstract}

Palabras clave: riesgos de la ciberconducta sexual, agresión sexual online, cibercortejo, adolescencia

\section{Cyberdating in adolescence: the risks and the emotional harm of sexual cyberbehavior}

\begin{abstract}
The new technologies have changed the way people interact with each other, especially among adolescents. The co-construction model stress that online and offline context are connected, so adolescents express the tasks and issues of their age, as sexuality and romantic relationships, in both contexts. These developmental tasks are an important source of learning, but some difficulties can appear, as sexual aggressions and other risky sexual behaviors. The attention to aggressive and risk of sexual behavior online has increased in the last years although results are not conclusive. This study will analyze the prevalence of
\end{abstract}


these behaviors among adolescents, into peer and couple context. The perceived emotional harm will be also analyzed. 268 adolescents with sentimental experience (mean age 14.22, s.d. 1.44) were interviewed using an adaptation of the Sexual Harassment Survey. Results showed that aggressive and risky sexual behaviors online were present at similar level in both, peer and couple context. The most frequent behaviors were visual/verbal sexual messages. Boys were more involved than girls for perpetration but no differences appear for receiving it. Girls felt more upset than boys after receiving these behaviors. Results are discussed in terms of the meaning of these behaviors for adolescents' social development.

Keywords: risks of sexual online behaviors, online sexual harassment, cyberdating, adolescence

\section{INTRODUCCIÓN}

La adolescencia, como periodo evolutivo marcado por importantes cambios físicos, cognitivos y psicosociales, sitúa a los jóvenes ante importantes retos o tareas psicoevolutivas, como la construcción de la identidad, la intimidad, la sexualidad o el inicio de las relaciones de pareja (Subrahmanyam y Smahel, 2011). La maduración biológica transformará los cuerpos infantiles en cuerpos adultos sexualmente maduros, y en este proceso, chicos y chicas tendrán que aprender a modular, expresar y vivir su sexualidad de acuerdo a las convenciones sociales y culturales, favoreciendo el inicio de las relaciones erótico-sentimentales y contribuyendo al desarrollo de su identidad. Aunque no es tarea fácil, lo cierto es que la mayoría de los adolescentes desarrollan con rapidez toda una serie de competencias, que bien aprendidas y utilizadas, les permitirán vivir y expresar su interés sexual de forma saludable. Sin embargo, un mal aprendizaje de éstas podría favorecer tanto la expresión desajustada como la vivencia de actitudes y comportamientos sexuales, molestos y desagradables.

Los estudios realizados hasta el momento revelan que estos comportamientos, que en la literatura internacional se denomina sexual harassment, se encuentran muy presentes en las redes de iguales adolescentes, con unas tasas de prevalencia que varían del 30\% al 84\% (Espelage, Basile y Hamburger, 2012; Ortega, Sánchez y Ortega-Rivera, 2008). Pese a que todavía existen lagunas respecto al constructo teórico y al desarrollo de instrumentos de medida lo cierto es que la violencia sexual en la adolescencia es considerada como un problema que perturba la salud pública (Bucchianeri, Eisenberg y Neumark-Sztainer, 2013) impactando directamente en el clima de los centros educativos (Lacasee, Purdy y Mendelson, 2003).

\section{La sexualidad adolescente en la era digital}

Ya nadie duda que internet y, en general, las nuevas tecnologías de la comunicación y la información han modificado de forma sustantiva el modo en que nos relacionamos especialmente entre los más jóvenes. El modelo de la co-construcción (Subrahmanyam y Smahel, 2011) defiende que para entender el desarrollo 
de los adolescentes actuales en su complejidad, se hace necesario partir de la asunción de que el mundo online y offline se encuentran íntimamente conectados. Desde esta perspectiva, la actividad de los jóvenes en la red expresaría los retos y tareas evolutivas que están afrontando en estos años, por lo que cabría esperar que las dificultades y riesgos que puedan encontrar en la gestión, vivencia y expresión de su sexualidad en la vida offline, se manifestasen también en el contexto online, aunque matizadas y moduladas por las características del propio contexto. Este es el caso del sexting (Lenhart, 2009), lo que antes quedaba en la esfera privada de las personas implicadas, como la pareja adolescente, ahora puede convertirse en viral en la red y ser visto por millones de personas en pocas horas.

Este estudio explorará los riesgos del cibercortejo y el cyberdating adolescente (Sánchez, Muñoz-Fernández y Ortega-Ruiz, 2015), entendido como todos aquellos comportamientos y actitudes de naturaleza sexual que los adolescentes despliegan en el medio online en sus interacciones con iguales y con sus parejas, y que pueden resultar agresivas o molestas, a lo que denominaremos agresiones y riesgos de la ciberconducta sexual (Livingstone y Smith, 2014). Incluye desde comportamientos y actitudes agresivas de naturaleza sexual, hasta conductas que, aunque per se no deberían ser consideradas como agresivas o violentas, como el sexting o el intercambio de pornografía, sí que pueden suponer un riesgo para el desarrollo de los adolescentes, y ser percibidas de forma violenta y molesta por quienes las reciben (Ringrose, Gill, Livingstone y Harvey, 2012).

La complejidad de comportamientos y miradas con las que se aborda el estudio de la ciberconducta sexual en la adolescencia ha provocado que se haya estudiado de forma desagregada. Desde el cyberbullying se ha prestado atención a las ciberagresiones sexuales como el envío de mensajes obscenos, denigrantes, imágenes pornográficas, e información con contenido íntimo y privado. Desde la perspectiva del riesgo que para los jóvenes supone la exposición a los mass media, se han focalizado en ciberconductas como el sexting, el grooming o la exposición de la pornografía (Livingstone y Smith, 2014). Una consideración que complejiza aún más el estudio del fenómeno tiene que ver con el malestar percibido por los adolescentes. Los estudios realizados en la pasada década sobre la violencia sexual cara a cara reflejaron cómo para algunos adolescentes, especialmente los chicos, muchos de estos comportamientos no eran percibidos como molestos o agresivos; mientras que para otros, fundamentalmente chicas, podían vivirlo como un verdadero problema, de forma intensa y personal (Timmerman, 2003; Menesini y Nocentini, 2008). En el contexto online los estudios apuntan en la misma dirección. Jones, Mitchell y Finkelhor (2012), preguntaron por el impacto emocional de la exposición a la pornografía, encontrando que un $50 \%$ de niños de 10 a 12 años y un $15 \%$ de 16 a 17 se sintieron disgustados por hacerlo. Respecto al sexting, los resultados del proyecto EukidsOnline encontraron que solo un $25 \%$ reportó sentirse molesto por haber recibido estos mensajes (Livingstone, Kirwil, Ponte, y Staksrud, 2014).

Los estudios sobre la prevalencia del fenómeno son todavía poco conclu- 
yentes, pues no se dispone a día de hoy de instrumentos de medida globales (Livingstone y Smith, 2014). Khurana, Bleakley, Jordan y Romer (2015), encontraron que aproximadamente el $25 \%$ de los adolescentes entrevistados manifestaron que les habían publicado en internet información íntima personal que no querían compartir y habían recibido mensajes ofensivos con contenido sexual, estando más implicadas las chicas que los chicos. Ellos sin embargo, han aparecido más implicados en el envío de mensajes con contenido sexual.

Por lo que respecta a los riesgos de la ciberconducta sexual, Baumgartner, Valkenburg y Peter (2010) encontraron que el 7.1\% de los adolescentes habían buscado a alguien para hablar de sexo y un $4.4 \%$ los habían buscado para tener sexo en internet. Las solicitudes sexuales, como pedir imágenes sexuales o hablar de sexo, ocurrieron en un $9 \%$ de adolescentes, siendo más frecuentes entre adolescentes mayores y en chicas (Jones et al., 2012).

Los estudios sobre sexting han mostrado tasas de prevalencia controvertidas, desde un $1 \%$ hasta un $40 \%$, dependiendo de la edad y conductas analizadas (Lenhart, 2009; Mitchell, Finkerhor, Jones y Wolak, 2012). En una revisión reciente, Klettke, Hallford y Mellor (2014), concluyeron que el 11.9\% de los adolescentes entre 10 y 19 años habían enviado alguna foto con contenido sexual o sugerente, siendo algo superior el porcentaje de quienes lo recibieron. Posteriormente, Khurana y colaboradores (2015), encontraron porcentajes cercanos al $25 \%$, aunque en su estudio se incluyeron también comportamientos como recibir mensajes sexuales molestos. En relación al sexo, algunos estudios han encontrado mayor implicación de las chicas (Mitchell et al., 2012), mientras que otros no han encontrado esta diferencia (Lenhart, 2009).

En el contexto de la pareja, Zweig, Dank, Lachman y Yahner (2013a) encontraron que entre un $4 \%$ y $22 \%$ de los adolescentes afirmaron haber recibido agresiones de naturaleza sexual de su pareja, siendo cercanos al 5\% los casos de agresión. Respecto al sexting, un $11.2 \%$ de los adolescentes dijeron haber recibido algún tipo de contenido sexual $-14.8 \%$ chicas y $7.2 \%$ chicos- y un $2 \%$ afirmó haberlas realizado $-3.8 \%$ chicos y $1.6 \%$ chicas (Zweig, Dank, Yahner y Lachman, 2013b).

En nuestro país, los estudios son todavía escasos y difícilmente comparables. Vilà, Donoso, Prados, Rubio y Velasco (2014) observaron que aproximadamente un $20 \%$ de los adolescentes habían enviado imágenes personales semidesnudos a otros sin su consentimiento, y un 39\% afirmó enviar mensajes sugerentes. Algo superiores fueron los porcentajes de los adolescentes que afirmaron haber recibido mensajes con contenido sexual no deseado, entre un 40\% y un 50\% (Montiel, 2014; Vilà et al., 2014). Respecto a las solicitudes, la encuesta de Unicef señaló que un $20 \%$ de las chicas y el $7 \%$ de los chicos afirmaron que alguna persona que habían conocido en internet le había pedido que les enviaran fotos semidesnudos (Fajardo, Gordillo y Regalado, 2013). Los estudios en pareja son muy escasos. Vilà et al (2014), encontraron tasas de sexting superiores a las encontradas en el contexto de los iguales: el 71\% y $67 \%$ (chicas y chicos respectivamente) enviaron imágenes o mensajes con contenido sexual a su pareja, siendo las chicas 
quienes consideraron más violenta la conducta "exhibir fotos sexis de la pareja sin permiso".

A partir de la literatura revisada, este estudio plantea el análisis de la prevalencia de las agresiones y riesgos de la ciberconducta sexual ejercidas y recibidas en la adolescencia. Asumiendo una perspectiva psicoevolutiva, en este análisis se contemplarán tanto las conductas de exploración e intercambio de material de contenido sexual, como las agresiones y riesgos de la ciberconducta - peticiones sexuales o el sexting- que pudiesen aparecer tanto entre iguales como en las parejas, analizando el efecto del sexo. Dada la escasez de estudios en nuestro país, se valorará también si los jóvenes que reciben estos comportamientos los perciben como molestos o desagradables.

\section{METODOLOGÍA}

\section{Participantes}

Participaron 268 adolescentes con experiencia sentimental previa, seleccionados a través de un muestreo intencional en centros educativos de Educación Secundaria Obligatoria de la provincia de Sevilla (España) en base a dos criterios: centros públicos y de áreas con nivel socio-económico medio. El rango de edad estuvo comprendido entre 12 y 18 años $(M=14.22$, d.e. 1.44$)$ siendo un $47.5 \%$ chicos y un $97.2 \%$ heterosexual. En el momento del estudio 82 adolescentes tenían pareja, con una duración media de la relación de 28.84 semanas (d.e. 21.62).

\section{Instrumentos}

Se utilizó una versión adaptada del Sexual Harassment Survey (American Association University Women, 1993) adaptada al contexto online. A través de 40 ítems (10 para agresión y 10 para victimización, para iguales y para parejas) medidos en escala likert de 5 puntos ( 0 nunca a 4 siempre), se preguntó a los adolescentes la frecuencia con la que habían realizado o sufrido agresiones y riesgos sexuales en el contexto online no deseados en los últimos seis meses. La consistencia interna fue buena, $\alpha=.89$ (recibidos-pareja); $\alpha=.89$ (ejercidospareja); $\alpha=.87$ (recibidos-iguales); $\alpha=.89$ (ejercidos-iguales).

Impacto emocional: con el objetivo de analizar el malestar emocional de cada comportamiento recibido se les preguntó “¿cómo te sentiste cuando te sucedió?" con opciones de respuesta desde 0 (no me importó) hasta 4 (me sentí muy mal).

\section{Procedimiento}

El instrumento fue administrado en horario escolar, en una sesión de 30 minutos, por investigadores entrenados. Se obtuvo consentimiento informado de los padres de los menores, y el anonimato y confidencialidad de la información 
estuvo garantizada.

\section{RESULTADOS}

El primer objetivo de este trabajo era explorar la presencia de la agresión y los riesgos de la ciberconducta sexual entre iguales y entre parejas. Dado el carácter descriptivo de este trabajo, y con el objetivo de favorecer la comparación con estudios previos, los datos de frecuencia se analizaron ítem a ítem.

Las respuestas se recodificaron en dos niveles, no implicados e implicados - quienes habían realizado o recibido el comportamiento en alguna ocasión-.

\section{Prevalencia de las agresiones y riesgos de ciberconducta sexual recibidas}

En la Tabla 1, se muestra la prevalencia de la victimización para cada comportamiento en función del sexo de los participantes en el contexto de los iguales y en la pareja. En los iguales, los comportamientos recibidos oscilaron entre un $15 \%$ y un $41 \%$ en los chicos, y entre un $10 \%$ y un $37 \%$ en las chicas. Para ellos y ellas, los comportamientos en los que estuvieron más implicados fueron "haber recibido bromas sexuales" ( $41 \%$ chicos y $37 \%$ chicas) y "haber recibido comentarios o gestos obscenos" (40\% chicos y $31 \%$ chicas). El comportamiento con menor implicación en ambos sexos fue "haber recibido comentarios obscenos sobre su comportamiento sexual" (15\% chicos y $10 \%$ chicas).

La prevalencia en la pareja osciló entre un $10 \%$ y un $45 \%$ en los chicos y entre un $4 \%$ y un $43 \%$ en las chicas. El comportamiento en el que hubo mayor implicación en ambos sexos fue "haber recibido bromas sexuales" (45\% chicos y $43 \%$ chicas), seguido de "haber hablado de sexo por internet" $43 \%$ chicos y $30 \%$ chicas). En comparación con los iguales, en la pareja casi se duplicaron los comportamientos, "haber recibido mensajes y fotos provocativas" (33\% pareja vs. $17 \%$ iguales) y "haber recibido peticiones de fotos o imágenes provocativas" (30\% pareja vs. $15 \%$ iguales).

Respecto al sexo, los análisis chi-cuadrado mostraron diferencias significativas en el ítem "haber hablado de sexo por internet", entre iguales y entre parejas. En ambos casos, en los chicos hubo mayor implicación que en las chicas. Entre parejas, hubo diferencias en los ítems "haber recibido rumores o comentarios sobre el comportamiento sexual", estando ellos tres veces más implicados que ellas, y en los ítems "hacer comentarios o gestos obscenos" así como en "enseñar, dar o dejar imágenes sexuales o mensajes obscenos", en los que ellos estuvieron aproximadamente dos veces más implicados que ellas. 
Tabla 1. Prevalencia y Malestar Emocional de las Agresiones y Riesgos de la Ciberconducta Sexual Recibidos por Iguales y por las Parejas en Función del Sexo (Datos en Porcentajes)

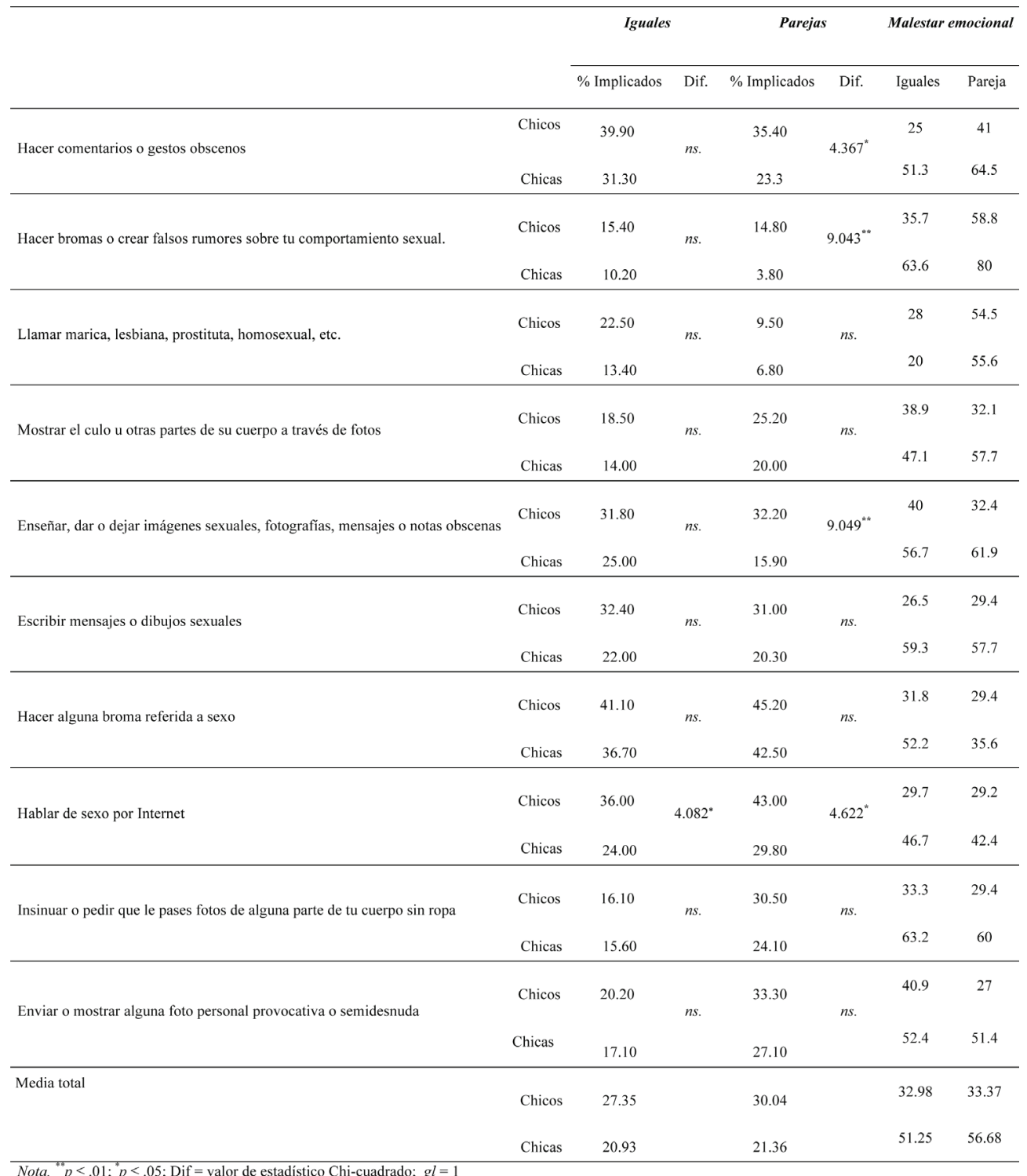




\section{Prevalencia de las agresiones y riesgos de ciberconducta sexual ejercidas}

En la Tabla 2, se presentan los datos de prevalencia de los comportamientos ejercidos. Entre los iguales los comportamientos con mayor implicación fueron "hacer comentarios o gestos obscenos" (33\% y 17\% para ellos y ellas), "hacer bromas sexuales" ( $36 \%$ chicos y $16 \%$ chicas), mientras que "insinuar o pedir que le pase fotos de alguna parte de tu cuerpo" fue el comportamiento con menor implicación. Entre parejas, los comportamientos con mayor implicación fueron "hablar de sexo" ( $40 \%$ y $23 \%$ para ellos y ellas) y "bromas sexuales" ( $37 \%$ chicos y $20 \%$ chicas). El comportamiento en el que hubo una menor implicación para ellos fue "llamar marica, lesbiana, prostituta, etc" (11\%) y para ellas "hacer bromas o crear falsos rumores sobre el comportamiento sexual" (1\%). El análisis entre contextos reveló que los comportamientos se ejercieron en porcentajes similares excepto para las conductas de sexting (i.e., "mostrar el culo u otras partes del cuerpo a través de fotos", "insinuar o pedir que le pases fotos de alguna parte de tu cuerpo sin ropa" o "enviar o mostrar alguna foto personal provocativa o semidesnuda"), donde hubo mayor implicación entre parejas.

Entre iguales, los chicos estuvieron significativamente más implicados que ellas en todas las conductas, excepto para el sexting. En las parejas, también los chicos estuvieron significativamente más implicados en todas las conductas, excepto en dos comportamientos de sexting ("insinuar o pedir que le pases fotos de alguna parte de tu cuerpo sin ropa" o "enviar o mostrar alguna foto personal provocativa o semidesnuda"), así como en "llamar marica, lesbiana, prostituta, etc" y "escribir mensajes sexuales" en los que no se encontraron diferencias significativas por sexo. 
Tabla 2. Prevalencia de las Agresiones y Riesgos de la Ciberconducta Sexual Ejercidas entre Iguales y en Parejas en Función del Sexo (Datos en Porcentajes)

\begin{tabular}{|c|c|c|c|c|c|}
\hline & & \multicolumn{2}{|c|}{ Iguales } & \multicolumn{2}{|c|}{ Parejas } \\
\hline & & $\%$ Implicados & Dif. & $\%$ Implicados & Dif. \\
\hline \multirow[t]{2}{*}{ Hacer comentarios o gestos obscenos } & Chicos & 33.60 & $8.676^{* \hbar}$ & 34.50 & $17.918^{\text {nikn }}$ \\
\hline & Chicas & 17.20 & & 12.10 & \\
\hline \multirow[t]{2}{*}{ Hacer bromas o crear falsos rumores sobre tu comportamiento sexual. } & Chicos & 13.40 & $9.115^{* *}$ & 11.30 & $12.570^{n+*+1}$ \\
\hline & Chicas & 2.50 & & 0.80 & \\
\hline \multirow[t]{2}{*}{ Llamar marica, lesbiana, prostituta, homosexual, etc. } & Chicos & 21.60 & $10.322^{* *}$ & 11.20 & $n s$ \\
\hline & Chicas & 7.20 & & 6.10 & \\
\hline \multirow[t]{2}{*}{ Mostrar el culo u otras partes de su cuerpo a través de fotos } & Chicos & 12.00 & $n s$. & 13.50 & $4.770^{*}$ \\
\hline & Chicas & 5.00 & & 5.40 & \\
\hline \multirow[t]{2}{*}{ Enseñar, dar o dejar imágenes sexuales, fotografias, mensajes o notas obscenas } & Chicos & 22.10 & $12.797^{* *}$ & 18.30 & $8.828^{* *}$ \\
\hline & Chicas & 6.30 & & 6.10 & \\
\hline \multirow[t]{2}{*}{ Escribir mensajes o dibujos sexuales } & Chicos & 26.60 & $11.089^{* *}$ & 20.40 & $n s$. \\
\hline & Chicas & 9.80 & & 12.80 & \\
\hline \multirow[t]{2}{*}{ Hacer alguna broma referida a sexo } & Chicos & 35.70 & $12.857^{\mathrm{kn}}$ & 36.60 & $8.493^{* *}$ \\
\hline & Chicas & 15.70 & & 19.80 & \\
\hline \multirow[t]{2}{*}{ Hablar de sexo por Internet } & Chicos & 30.30 & $6.092^{*}$ & 39.50 & $7.670^{\text {** }}$ \\
\hline & Chicas & 16.80 & & 23.10 & \\
\hline \multirow[t]{2}{*}{ Insinuar o pedir que le pases fotos de alguna parte de tu cuerpo sin ropa } & Chicos & 7.20 & $n s$. & 14.00 & $n s$. \\
\hline & Chicas & 2.40 & & 8.30 & \\
\hline \multirow[t]{2}{*}{ Enviar o mostrar alguna foto personal provocativa o semidesnuda } & Chicos & 9.20 & $n s$. & 15.90 & $n s$. \\
\hline & Chicas & 5.40 & & 10.60 & \\
\hline \multirow[t]{2}{*}{ Media total } & Chicos & 21.17 & & 21.52 & \\
\hline & Chicas & 8.83 & & 10.51 & \\
\hline
\end{tabular}




\section{El impacto emocional percibido}

Respecto al impacto emocional, la Tabla 1 presenta los porcentajes de chicos y chicas que afirmaron sentirse molestos tras recibir cada uno de los comportamientos analizados. Estos porcentajes se calcularon a partir de quienes afirmaron estar implicados, dicotomizando la respuesta en 0 , quienes no les había importado, y 1, a quienes les había molestado desde poco hasta mucho. Entre un $20 \%$ y un $80 \%$ de los participantes que afirmaron haber recibido algún comportamiento, se sintieron molestos, siendo el malestar mayor cuando se recibía de la pareja en los casos de insultos sexuales y menor en el sexting, en ellos. Respecto al sexo, las chicas se sintieron más molestas que ellos en todos los ítems excepto en "te han llamado lesbiana, marica, homosexual, prostituta", donde ellos presentaron niveles algo superiores.

\section{CONCLUSIONES}

El objetivo del estudio fue explorar las agresiones y riesgos de la ciberconducta sexual en la adolescencia desde una perspectiva psicoevolutiva. Desde este enfoque, se analizaron ciberconductas relacionadas con los intercambios de mensajes e imágenes sexuales, agresiones verbales sexuales y conductas de sexting, tanto entre iguales como entre parejas adolescentes.

El análisis de los comportamientos concretos ha permitido constatar que los más frecuentes, recibidos y ejercidos, entre iguales y entre parejas tienen que ver con bromas sexuales, comentarios obscenos y hablar de sexo, lo que confirma la necesidad de exploración sexual en los años adolescentes (Subrahmanyam y Smahel, 2011), alcanzando tasas de prevalencia en torno al 30\%, en línea con estudios internacionales (Khurana et al., 2015) y nacionales (Vilá et al., 2014). Los insultos sexuales recibidos y ejercidos fueron menos frecuentes en ambos contextos, y especialmente en las chicas, con tasas de implicación media que no superaron el 7\%. Finalmente, enviar y pedir imágenes personales provocativas fue más frecuente entre parejas que entre iguales, en los comportamientos recibidos y realizados, lo que confirma que una de las principales motivaciones para implicarse en este tipo de comportamientos tiene que ver con demostrar interés sexual (Henderson y Morgan, 2011; Vilá et al., 2014).

Las tasas de implicación encontradas entre iguales han sido similares a las de estudios previos: algo menos de dos de cada diez adolescentes afirmaron haber recibido fotos o peticiones de sus iguales, y algo menos de 1 de cada diez afirmó haberlo realizado (Jones et al., 2012). Los datos en el contexto de la pareja han resultado, sin embargo, inferiores a los encontrados en estudios nacionales, en comportamientos recibidos y realizados (Vilá et al., 2014), pero cercanos a los datos internacionales (Zweig et al., 2013b), lo que podría estar explicado por los diferentes instrumentos de medida utilizados.

El sexo ha mostrado tener un efecto diferencial en la ciberconducta sexual, fundamentalmente para los comportamientos ejercidos, aproximadamente $2 \mathrm{de}$ 
cada diez chicos y una de cada diez chicas afirmaron haber enviado algún comentario, gesto o imagen provocativa en los últimos seis meses a su grupo de iguales y a sus parejas. Estos datos son coherentes con estudios previos donde las chicas realizan menos estos comportamientos tanto en el contexto online ( $\mathrm{Li}$ vingstone, et al., 2013) como offline (Ortega, Sánchez y Ortega-Rivera, 2008), contrastando con los estudios que han concluido que ellas reciben más estas ciberconductas (Khurana et al., 2015). En este sentido, en líneas generales nuestros datos no han mostrado diferencias significativas en el sexting en ninguno de los contextos analizados, de acuerdo al estudio de Lenhart (2009), pero en contra de lo encontrado en otros trabajos (Khurana, et al., 2015). El tipo de comportamientos concretos que cada estudio ha analizado podría estar explicando estas diferencias, haciéndose necesario que la comunidad científica aporte una definición consensuada respecto a estos fenómenos.

El análisis del impacto emocional ha presentado resultados clarificadores respecto a la percepción que los adolescentes tienen de estos comportamientos. En general, entre un $30 \%$ de chicos y un $50 \%$ de chicas afirmaron sentirse molestos. Estos resultados son coherentes con estudios anteriores en el medio online (Ringrose et al., 2012) y offline (Menesini y Nocentini, 2008), en el sentido de que muchos adolescentes no perciben estos comportamientos como molestos o violentos, justificando su implicación. Es importante, sin embargo, matizar que para ellas estos comportamientos eran más molestos, lo que refleja la vivencia diferencial de la sexualidad entre sexos; es probable que para ellos recibir estos comportamientos sea algo positivo y que refuerce su masculinidad, y para ellas sea una invasión de su intimidad y privacidad (Ortega, Ortega-Rivera y Sánchez, 2008), entendiéndose así por qué ellos sólo se sintieron más molestos cuando les "llamaron marica, lesbiana, o prostituta", pues incide directamente en su masculinidad. No conocemos estudios previos que hayan comparado el malestar asociado a estas conductas en el contexto de iguales y en la pareja, pero nuestros resultados apuntan a que en la pareja los insultos sexuales son mucho más molestos que entre los iguales, probablemente por la vinculación afectiva de la diada. Futuros estudios podrían profundizar en las razones por las que los adolescentes consideran estas conductas como molestas o no.

Este trabajo ha pretendido analizar los riesgos del cibercortejo y el cyberdating desde una perspectiva psicoevolutiva, vinculando las agresiones y los riesgos de la ciberconducta sexual a una de las principales tareas evolutivas de estos años: la sexualidad. Aunque el número de participantes ha sido pequeño y la naturaleza del estudio ha sido exploratoria, los resultados han revelado que estas conductas están presentes en aproximadamente dos de cada diez adolescentes en el medio online, tanto entre iguales como entre parejas. Aunque los datos son algo inferiores a los encontrados en los estudios cara a cara, confirman la interconexión entre los contextos, demandando la necesidad de incluir ambos escenarios en el estudio de los riesgos de la conducta sexual y en el desarrollo de programas de prevención. 


\section{AGRADECIMIENTOS}

Esta investigación ha sido financiada por el Programa Estatal de Investigación, Desarrollo e Innovación orientada a los Retos de la Sociedad (I+D+i 20132016) "Parejas y Redes de iguales en la adolescencia" (PSI2013-45118-R). La segunda autora disfruta de un contrato predoctoral FPU,convocatoria 2013 del Ministerio de Educación, Cultura y Deporte.

\section{REFERENCIAS}

American Association of University Women. (1993). Hostile hallways: The AAUW survey on sexual harassment in America's schools. Washington, DC: American Association of University Women.

Baumgartner, S. E., Valkenburg, P. M., y Peter, J. (2010). Assessing causality in the relationship between adolescents' risky sexual online behavior and their perceptions of this behavior. Journal of youth and adolescence, 39(10), 12261239.

Bucchianeri, M. M., Eisenberg, M. E., y Neumark-Sztainer, D. (2013). Weightism, racism, classism, and sexism: Shared forms of harassment in adolescents. Journal of Adolescent Health, 53(1), 47-53

Espelage, D. L., Basile, K. C., y Hamburger, M. E. (2012). Bullying perpetration and subsequent sexual violence perpetration among middle school students. Journal of Adolescent Health, 50, 60-65.

Fajardo, M. I., Gordillo, M., y Regalado, A. B. (2013). Sexting: Nuevos usos de la tecnología y la sexualidad en adolescentes. International Journal of Developmental and Educational Psychology, 1(1), 521-534.

Henderson. L, y Morgan. E. (2011). Sexting and sexual relationships among teens and young adults. McNair Scholars Research Journal, 7(1), 31-39.

Jones, L. M., Mitchell, K. J., y Finkelhor, D. (2012). Trends in youth internet victimization: Findings from three youth internet safety surveys 2000-2010. Journal of Adolescent Health, 50(2), 179-186.

Khurana, A., Bleakley, A., Jordan, A. M., y Romer, D. (2015). The Protective Effects of Parental Monitoring and Internet Restriction on Adolescents' Risk of Online Harassment. Journal of Youth and Adolescence, 44(5), 1039-147.

Klettke, B., Hallford, D. J., y Mellor, D. J. (2014). Sexting prevalence and correlates: a systematic literature review. Clinical psychology review, 34(1), 44-53.

Lacasse, A., Purdy, K., y Mendelson, M. (2003). The mixed company they keep: Potentially offensive sexual behaviours among adolescents. International Journal of Behavioral Development, 27(6), 532-540.

Lenhart, A. (2009). Teens and Sexting: How and why minor teens are sending sexually suggestive nude or nearly nude images via text messaging. Washington, D. C.: Pew Internet y American Life Project.

Livingstone, S., Kirwil, L., Ponte, C., y Staksrud, E. (2013). In their own words: 
what bothers children online? with the EU Kids Online Network. London: London School of Economics y Political Science.

Livingstone, S., y Smith, P. K. (2014). Annual Research Review: Harms experienced by child users of online and mobile technologies: the nature, prevalence and management of sexual and aggressive risks in the digital age. Journal of child psychology and psychiatry, 55(6), 635-654.

Menesini, E., y Nocentini, A. (2008). Comportamenti aggressivi nelle prime esperienze sentimentali in adolescenza. Giornale Italiano di Psicologia, 2, 407- 434.

Mitchell, K. J., Finkelhor, D., Jones, L. M. y Wollak. J. (2012). Prevalence and characteristics of youth sexting: a national study. Pediatrics, 129, 13-20.

Ortega, R., Ortega-Rivera, J., y Sánchez, V. (2008). La violencia sexual entre compañeros/as y en la pareja: un estudio exploratorio. International Journal of Psychology and Psychological therapy, 8, 63-72.

Ringrose, J., Gill, R., Livingstone, S., y Harvey, L. (2012). A qualitative study of children, young people and 'sexting': A report prepared for the NSPCC. London: NSPCC.

Subrahmanyam, K., y Smahel, D. (2011). Digital Youth: The Role of Media in Development. New York: Springer.

Timmerman, G. (2003). Sexual harassment of adolescents perpetrated by teachers and by peers: An exploration of the dynamics of power, culture, and gender in secondary schools. Sex Roles, 48(5-6), 231-244.

Vilà-Baños, R., Donoso-Vázquez, T., Prado, N., Rubio-Hurtado, M. J., y Velasco-Martínez, A. (2014). Investigando sobre violencias de género 2.0. En T. Donoso-Vázquez (coord.),Violencias de gnero 2.02.(pp.29-38). Barcelona: Kit-book.

Zweig, J. M., Dank, M., Lachman, P., y Yahner, J. (2013a). Technology teen dating violence and abuse, and bullying. Washington: Urban Institute, Justice Policy Center

Zweig, J. M., Dank, M., Yahner, J., y Lachman, P. (2013b). The rate of cyber dating abuse among teens and how it relates to other forms of teen dating violence. Journal of Youth and Adolescence, 42(7), 1063-1077. 